\title{
Anti-Windup Techniques Applied to Buck-Boost Converter in Photovoltaic Systems: a Comparative Study
}

\author{
Raul Brito Cavalcante da Silva * Tito Luís Maia Santos ** \\ * Department of Electrical Engineering, Programa de Pós-Graduação \\ em Engenharia Elétrica, Federal University of Bahia, BA, (e-mail: \\ raulbc40@gmail.com,**tlsantos@ufba.br).
}

\begin{abstract}
This work presents an analysis of irradiance and temperature variation effect applied to a solar panel linked by a Buck-Boost converter concerning duty-cycle limits with a PID controller. The study is done numerically with a simulation software using three anti-windup strategies and real data of irradiance and temperature measured by Sistema de Organizacao Nacional de Dados Ambientais/Instituto Nacional de Pesquisas Espaciais (SONDA/INPE). The PID tuning is based on the root locus method for design simplicity with predefined specifications of overshoot and settling time without loss of generality. It is shown that modified anti-windup techniques improve closed-loop performance significantly when radiation decreases due to cloud effect.
\end{abstract}

Keywords: Windup, Irradiance, Saturation, Smart Grids, PID controllers.

\section{INTRODUCTION}

Photovoltaic solar energy and its expansion have been shown as viable alternatives to supply the growth in energy demand which occurs in national and international level. Besides being a renewable and intermittent source, this is non-polluting, silent energy that has been reducing its costs over the years (EIA, 2018). Moreover, solar energy conversion into electrical energy happens through the solar cell, which is made up of semiconductor materials with mono or polycrystalline silicon. Solar panels provide a wide range of output voltage and current. However, a set of defined values for the connected load, cell temperature, and solar irradiance can produce maximum power. The characteristic, voltage versus current, depends on the solar irradiance and the temperature of the photovoltaic cell, and these variables can cause fluctuations in the delivery of power to the load. Thus, controlled static converters are allocated between photovoltaic generators and loads, to keep the system working continuously within the range of operation of the chosen topology (Belhachat et al., 2007).

PID controllers are used in large-scale, mainly in the industrial area, due to some advantages such as vast literature on the subject, simplicity, robustness and low cost. Astrom and Rundqwist (1989) discuss the saturation effect on the actuator and its consequences in the feedback loop when the output signal from the control and input of the plant reaches undesirable values. Also, as this effect was seen in the integral term of the PID controller, this was called the windup integrator at first.

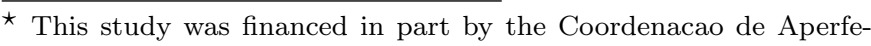
icoamento de Pessoal de Nivel Superior - Brazil (CAPES) - Finance Code 001.
}

The work done by Bernstein and Michel (1995) illustrates the chronological order of this study of the saturation action of actuators and windup over the years. Hippe (2006) shows the use of linear tools for non-linear models and expands the anti-windup analysis for systems with multiple inputs and outputs (MIMO). At the end of the $60 \mathrm{~s}$, early anti-windup studies inspected some algorithms for digital control over the integral action of PI and PID controllers (Fertik and Ross, 1967). In Hanus, a new tracking technique was proposed which was called back-calculation, where the signal output of the control follow the saturation limits and adds an extra feedback signal to the input of the integrator (Hanus, 1980). Furthermore, several other works were developed, and among them, the study of Hanson, Gruber, and Todtli on the Fuzzy logic implemented for PID controllers (Hansson et al., 1994). The work of Zheng et al. (1994) evaluates the basics of the internal model and which has been expanded by Soares with resonant, multiresonant and repetitive controllers (SOARES et al., 2010). Bohn and Atherton (1995) presented the first simulations with the Simulink software showing the techniques of backcalculation, conditional integration, and their variations. Hodel and Hall (2001) proposed a variable PID structure to prevent the phenomenon, which served for continuous and discrete-time situations.

Anti-windup techniques have been used in the context of converter's control, which cannot be found frequently in the presence of irradiance, temperature or other disturbances that cause saturation specifically. In Olalla et al., an innovative study of robust $H_{\infty}$ control was applied to a buck converter in continuous conduction mode (CCM), with automatic adjustment of controller gains and better ways of insertion of uncertainties, as well as ensuring a large margin of stability throughout the process (Olalla et al., 2009). Villalva et al. (2010) show advances in the 
way of working with solar panels, their regulation and their control working with a buck converter. Also, in Xiao et al. (2012) the peculiarities of the boost converter are shown for simulations with and without anti-windup strategies, involving non-linearities and the presence of a non-minimal phase in its transfer function. Finally, other contributions involving the anti-windup have been used with $\mathrm{K}$ factor for the design of controllers and a method of regulating voltage and frequency in the buck and buck-boost converters (Balamurugan and Selvaperumal, 2016).

This work analyzes the importance of modified antiwindup strategies in photovoltaic voltage regulation based on a buck-boost converter for cloud environments. A comparative study is presented to illustrate the advantages and drawbacks of different anti-windup schemes to mitigate the undesired irradiance variation effect in the context of voltage regulation based on buck-boost converters. Real irradiance and solar data are used in this comparative study which can be easily extended to other regions and weather scenarios.

The paper is organized as follows. Section 2 presents general control principles of buck-boost converters. Section 3 shows the PID tuning applied to a voltage closed loop with a converter and anti-windup strategies. Simulations of case studies are shown in section 4 to see the irradiance and temperature influence in this converter. Finally, conclusions are presented in section 5 .

\section{BUCK-BOOST CONVERTER MODELING}

The Buck-Boost converter schematic diagram is shown in Fig. 1. This converter presents a reverse diode in its structure and can operate in buck mode and boost mode. Also, the output voltage is negative to the ground Rashid et al. (2011). The buck-boost transfer function has a non-minimal phase zero as expressed in (1) by using an averaging method and small-signal linearization. Furthermore, the non-minimal phase zero is represented in (2) by $\omega_{z}, D^{\prime}=1-D$, quality factor in (3) by $Q_{2}$ and undamped natural frequency in (4) by $\omega_{02}$ (Erickson and Maksimovic, 2007).

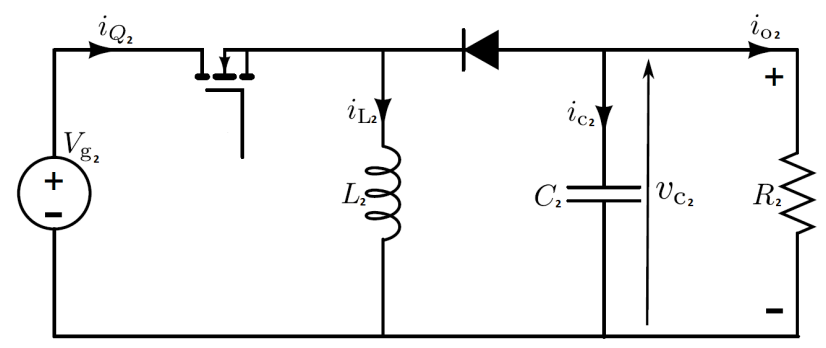

Figure 1. Buck-Boost Converter

$$
\begin{gathered}
G_{v 2}(s)=\frac{V_{g 2}\left(1-\left(\frac{s}{\omega_{z}}\right)\right)}{D^{\prime 2}\left(\left(\frac{s}{\omega_{02}}\right)^{2}+\left(\frac{s}{\omega_{02} Q_{2}}\right)+1\right)} \\
\omega_{z}=\frac{R_{2} D^{\prime 2}}{D L_{2}}
\end{gathered}
$$

$$
\begin{gathered}
Q_{2}=D^{\prime} R_{2} \sqrt{\frac{C_{2}}{L_{2}}} \\
\omega_{02}=\frac{D^{\prime}}{\sqrt{L_{2} C_{2}}}
\end{gathered}
$$

The DC voltage transfer function of a buck-boost circuit is shown as follows, confirming the reverse polarity of the output voltage:

$$
K_{v 2}=\frac{V_{\text {out }}}{V_{g 2}}=\frac{-D}{1-D}
$$

In (5), it is noticed that buck-boost converters can stepdown or step-up the voltage in your terminals, but it depends on the duty-cycle stage. If $0<D<0.5$, the converter operates in buck mode. Otherwise, if $0.5<D<$ 1 , buck-boost converter works as a boost converter.

\section{PID TUNING WITH ROOT LOCUS METHOD}

In this work, a continuous-time PID controller in parallel form has been designed and tuned with filtered derivative action. Furthermore, the tuning method used is the rootlocus method, which is a graphical method for sketching the locus roots of a transfer function in the s-plane, measuring the parameters of a PID controller and providing stability (Dorf and Bishop, 2011). In (6), $K_{p}$ is the proportional gain, $K_{i}$ is the integral gain, $K_{d}$ is de derivative gain and $N$ is the parameter of the filter of the derivative part.

$$
G_{c}(s)=K_{p}+\frac{K_{i}}{s}+\frac{K_{d} s}{N s+1}
$$

With the desired specifications and the transfer function of the converter, the dominant pole has to be proposed in order to choose and position a pair of conjugate poles which represent the requirements on a closed-loop response, such as steady-state error, settling time, rise time and overshoot (Wang et al., 2008). In the next section, these specifications are introduced and the controller will be found by the root locus.

Also, other tuning strategies can be used since the analysis can be performed without loss of generality since the cloud scenario would cause saturation with any control strategy. Moreover, note from (1) that the nominal linearized transfer function depends on $V_{g 2}$ which is a consequence of the solar irradiation and also depends on the duty-cycle.

\subsection{Voltage Loop Controller of Buck-Boost Converter}

The transfer function of a buck-boost converter is represented by (7), and there is a non-minimal phase zero. Table 1 shows the parameters and specifications to find the PID controller, derived by (8). 
Table 1. DC/DC BUCK-BOOST CONVERTER SPECIFICATION

\begin{tabular}{ccc}
\hline \hline Input Voltage & $V_{g 2}$ & $64 \mathrm{~V}$ \\
Output Voltage & $V_{c 2}$ & $-48 \mathrm{~V}$ \\
Load Resitance & $R_{2}$ & $10 \Omega$ \\
Inductance & $L_{2}$ & $8 m H$ \\
Capacitance & $C_{2}$ & $1 m \mathrm{~F}$ \\
PWM Switching Frequency & $f_{s}$ & $10 k H z$ \\
Overshoot & $M_{p}$ & $<20 \%$ \\
Settling Time & $T_{s}$ & $<0.1 s$ \\
\hline \hline
\end{tabular}

$$
\begin{gathered}
G_{v 2}(s)=\frac{-8400(s-952.4)}{s^{2}+100 s+4.08 \times 10^{4}} \\
C_{2}(s)=0.0077+\frac{0.47}{s}+\frac{0.00003 s}{0.00038 s+1}
\end{gathered}
$$

This PID controller could eliminate the overshoot and fix the settling time at 0.1 seconds. The influence of the nonminimal phase zero generates an undershoot in the step response.

\subsection{Anti-Windup Techniques}

The saturation action in control systems is related directly to the integral action. This effect occurs when integration becomes a continuous sum while the error consists in accumulating over time. In power converters, the duty cycle is the output of the controller, and it has to be limited from 0 to 1 . The windup, in general, appears when the controller assumes a linear operation, but cannot affect the controlled variable due to a saturation duty cycle (Xiao et al., 2012). Thus, the integral term of a PID controller may become very large, which can cause large overshoots and high settling times (Hippe, 2006).

Modified Conditional Integration: Fertik and Ross (1967), Bohn and Atherton (1995) and Rundqwist (1990) presented classical methods of this technique, which can switch on or off the integral term depending on certain conditions. In this paper, the modified conditional integrator will switch on or off based on buck-boost converter duty-cycle required, which can be achieved by (5). There is a conditional comparison; if $u \geq D$ then the integral term is suspended; otherwise, it will work usually. In Fig. 2, a block diagram shows this technique.

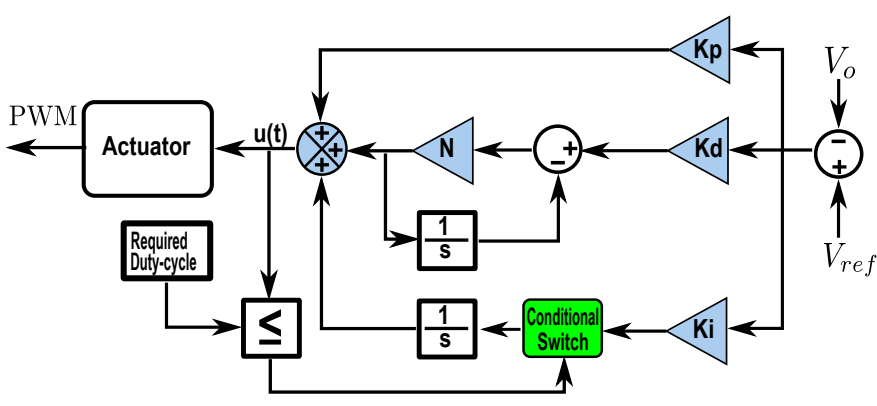

Figure 2. Anti-windup modified conditional integration technique with PID

Chen's Technique: The basic idea in this strategy is to disable the integrator when the signal $u(t)$ reaches the saturation level (Chen, 1993). In Fig. 3, if $|u(t)|<$ sat the input of integral term $w(t)=1$ and there is no influence in the integral term, otherwise if $|u(t)|>$ sat the input of integral term $w(t)=0$ and then occurs the windup prevention.

Relay block works with positive and negative values. If its input receives a positive value, then its output will be a binary number 1 . Otherwise, this output will be a binary number 0 .

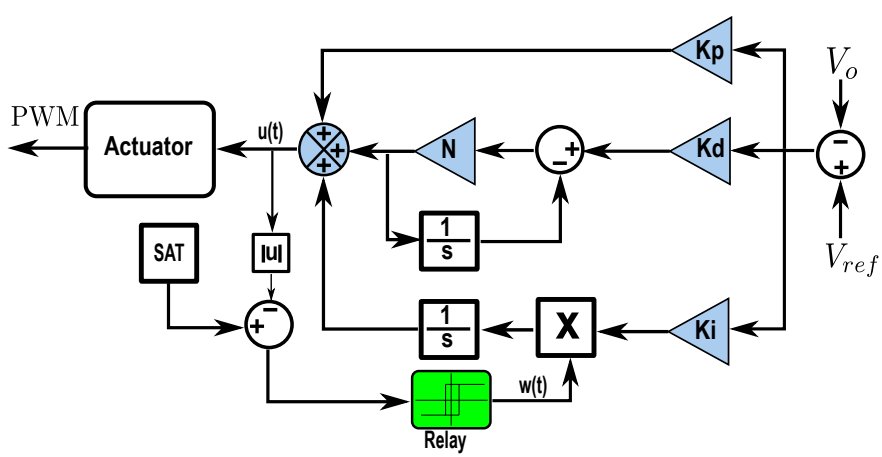

Figure 3. Chen's strategy with PID controller

Dead Zone: This technique aims to feed the integrator with a high gain to reduce the integrator input in the saturation cases (Bohn and Atherton, 1995). In Fig. 4, it shows that the strategy presents a negative feedback in the integral term with a tracking time constant defined as $K_{t}=\sqrt{K_{d} K_{i}}$.

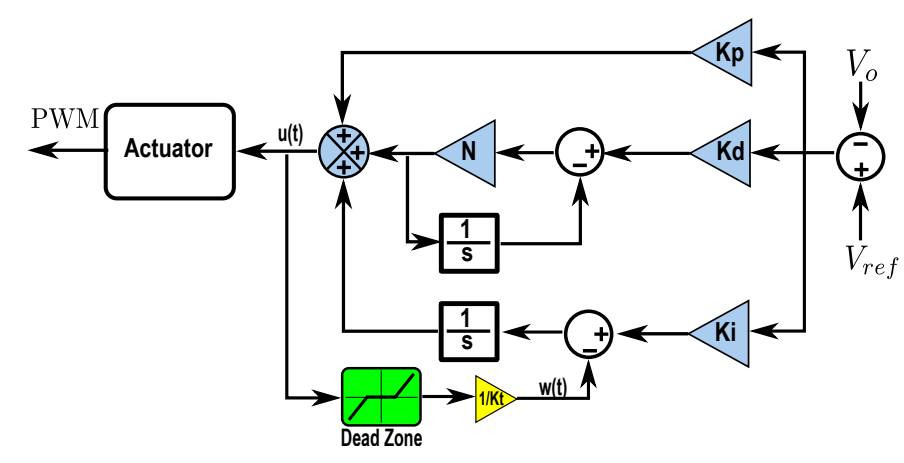

Figure 4. Dead Zone strategy with PID controller 
The dead zone limits are $Z_{\text {min }}$ and $Z_{\max }$, which can be the saturation limits of duty-cycle. This way, when $u(t)<|Z|$ there is no saturation, and the integral term will receive zero feedback due to the dead zone, so its signal control will remain unchanged. Diversely, if when $u(t)>|Z|$ then the dead zone is extrapolated and $u(t)$ is proportionally feedback in the integral term, which can attenuate this portion of PID control. Equation (9) shows how the dead zone works.

$$
w(t)= \begin{cases}0, & \text { if }|u|<Z \\ \frac{1}{K_{t}} \times\left(u-Z_{\max }\right) & \text { if } u \geq Z_{\max } \\ \frac{1}{K_{t}} \times\left(u+Z_{\min }\right) & \text { if } u \leq Z_{\min }\end{cases}
$$

In this work, a modified anti-windup concept is used to avoid sudden control variations in order to mitigate the undesired nonlinear effect. The main idea is to define antiwindup parameters in order to avoid an abrupt duty-cycle variation in the presence of significant disturbances.

\subsection{Modeling of a Solar Photovoltaic Array}

It is necessary to know how the circuit of a solar cell, in Fig. 5 , tends to behave in the presence of these variables to understand the behavior of a solar panel regarding irradiance and temperature variations. Also, this simplified model was purposely used to show how a simple perturbation of solar irradiance can compromise the performance of the output voltage even with a boost converter Buck-Boost type.

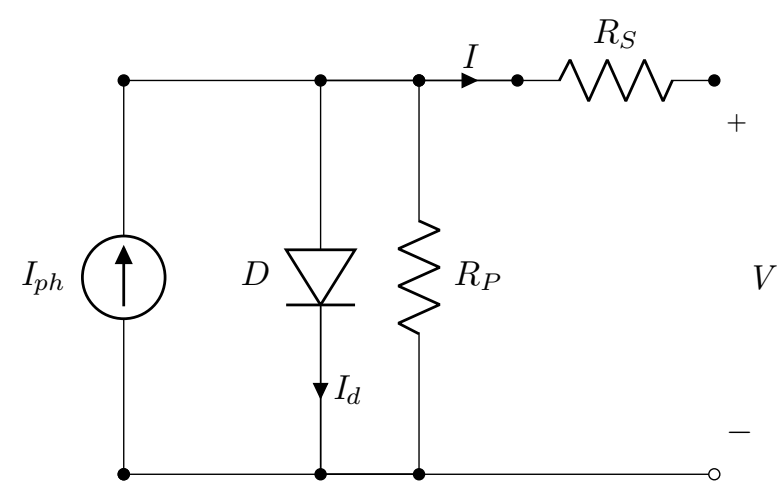

Figure 5. Photovoltaic cell circuit

The output cell current depends on output voltage $V$, irradiance $S$ and temperature $T$. Equation (10) is given by Rashid et al. (2011); Seghir et al. (2018); Cavalcanti et al. (2007),

$$
I=I_{p h}-I_{0} \cdot\left[e^{\frac{q \cdot\left(V+I . R_{S}\right)}{n . k . T}}-1\right]-\frac{V+I . R_{S}}{R_{P}}
$$

where $I_{p h}$ is the photocurrent, $I_{0}$ is the reverse saturation current, $I_{d}$ is diode current, $R_{P}$ is the shunt resistance, $R_{S}$ is the series resistance, $q$ is the electron charge, $k$ is the Boltzmann constant and $T$ is the solar cell temperature. In addition, the photocurrent is derived by (11),

$$
I_{p h}=\left[I_{s c}+\alpha_{T} \cdot\left(T-T_{r}\right)\right] \cdot \frac{S}{1000}
$$

where $I_{s c}$ is the short-circuit current, $\alpha_{T}$ is the shortcircuit temperature coefficient and $T_{r}$ is the temperature reference.

\subsection{Implementation}

Real irradiance and temperature data, in Fig. 6, of Brasilia-Brazil, on the first day of November from 14 hours and 47 minutes to 15 hours and 16 minutes of the 2015 year, was used in a solar panel with a buck-boost converter to see the influence of these disturbances at the saturation (SONDA, 2018).

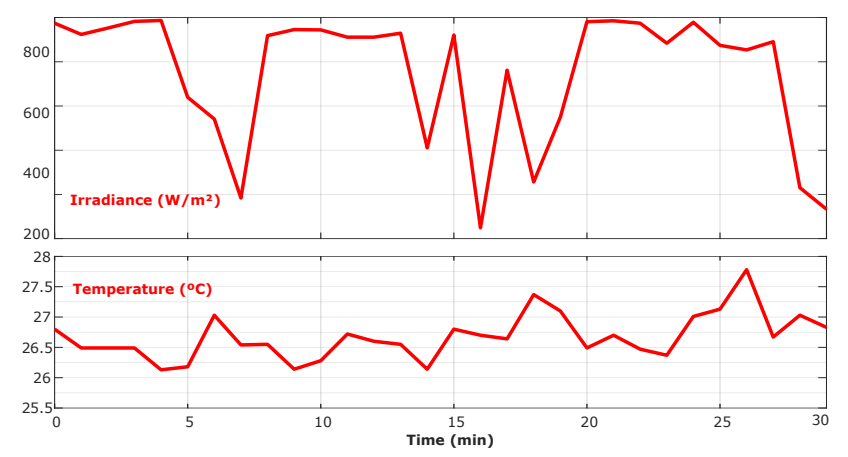

Figure 6. Real data of irradiance and temperature of Brasilia-Brazil

Three strategies were tested with a buck-boost converter. The solar panel model, converter and PID anti-windup controllers have been implemented on Simulink/Matlab software. The implementation can be seen in Fig.7.

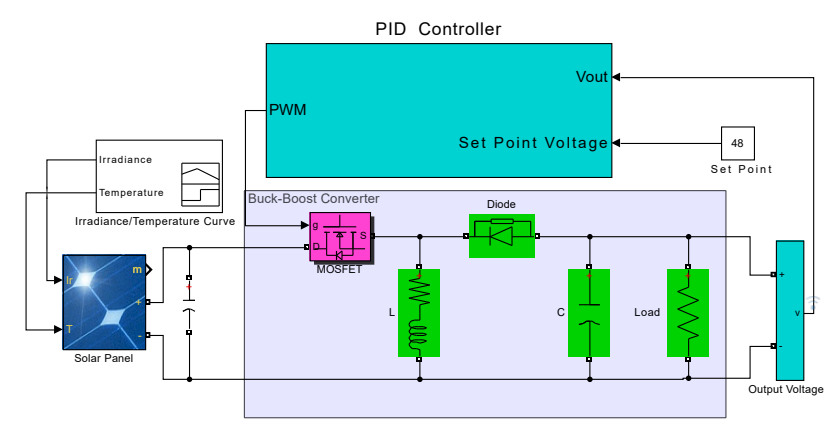

Figure 7. Schematic simulation diagram

Besides, solar panel specification is shown in the Table 2.

\section{Table 2. SOLAR PANEL ELECTRICAL SPECIFICATION}

\begin{tabular}{ccc}
\hline \hline Maximum Power & $P_{\max }$ & $235 \mathrm{Wp}$ \\
Maximum Power Current & $I_{m p}$ & $8.02 \mathrm{~A}$ \\
Short-Circuit Current & $I_{s c}$ & $8.54 \mathrm{~A}$ \\
Maximum Power Voltage & $V_{m p}$ & $30.8 \mathrm{~V}$ \\
Open Circuit Voltage & $V_{o c}$ & $37 \mathrm{~V}$ \\
\hline \hline
\end{tabular}




\section{SIMULATION CASE STUDIES}

In this section, the results of the output control signal and the output voltage are compared with each technique after the action of irradiance and temperature data. All tests consider a tolerance error of $1 \mathrm{e}-6$ for a normal simulation. Furthermore, two solar panels in series reach almost 62 volts of the input voltage in each case. The required dutycycle for modified conditional integrator was defined by 0.428 , according to specifications in Table 1 . The tracking time constant $1 / K_{t}$ was defined as 527 for buck-boost in dead zone's by using the inverse of $K_{t}=\sqrt{K_{d} K_{i}}$. The value of sat in Chen's technique basically must be used to identify if there is or there is not saturation, also verifying if the limit was reached and it was defined as 0.43 , which is the limit found to stabilize the duty-cycle and stabilize the output voltage. Moreover, at the dead zone technique the limits can be chose as $Z_{m i n}=0$ and $Z_{m x}=0.49$, also finding the better behavior to this case. Fig. 8 shows the results of the control signal when the buck-boost converter operates without anti-windup techniques and with those three strategies showed before.

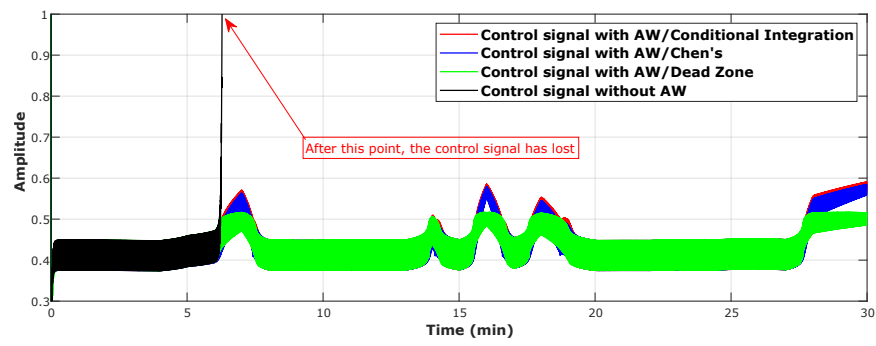

Figure 8. Control signal of a buck-boost converter with anti-windup strategies

Figure 8 can show when the irradiance decreases at the time between 6 and 7 minutes occurs the appearance of clouds and the duty-cycle reaches the limit due to irradiance and the voltage drop in the solar panel. Additionally, when the irradiance retrieves the value, the accumulation of the integral action is harmful, but it can be fixed with anti-windup action. The response of the control signal without anti-windup action confirms that the duty-cycle achieves levels higher than 1 . On the contrary, a modified conditional integrator strategy could attenuate this problem significantly. Next, Chen's strategy can eliminate some undesired values for a duty-cycle, and its behavior was good cause it is not attaining the superior limit. Equally, the dead zone technique caught up the required conduct of the control by not trespassing or not even oscillating when this action acts on the buck-boost converter. Thus, all three strategies had achieved the desired performance.

Following this, Fig. 10 presents the results of the output voltage of buck-boost converter without and with modified conditional integration, Chen's and dead zone techniques. The converter is exclusively intended for the control of the output voltage, in which there is no need to include any maximum power-point tracking (MPPT) technique in the control of the buck-boost converter. Also, it is important to notice that the voltages shown in the graph and for control purposes are given by the inverse of the measured output $\left(-V_{\text {out }}\right)$. The input voltage $V_{g 2}$ should work at the range between 64 and 48 volts because in this range the converter is in buck mode. If this voltage drops below 48 volts, the converter enters the boost mode, but the control signal is lost in this converter behavior change due to the undershoot in the buck-boost transfer function and can not raise the voltage to the reference while irradiance decreases.

It is shown that when the buck-boost converter works without any anti-windup method, the output voltage presents an offset caused by capacitor discharge, putting the output voltage at zero after minute 6 , which is undesirable. However, the modified conditional integrator can eliminate this problem; the voltage is still keeping a little retard and a little overshoot of $4 \%$ with respect to its set point, obeying the specifications. Besides, in Chen's, the signal control obey the limits in Fig. 8 and this overshoot is less than $3 \%$, which is desirable. Finally, the dead zone pursues the set point with $1 \%$ of overshoot, which is confirmed by the stabilized duty-cycle in Fig. 8. In addition, other classical technique was tested, which is called back-calculation, but its behavior was not able to avoid these clouds influences on buck-boost.

The anti-windup parameters were purposely chosen to prevent system behavior from deviating from the linearity zone. Thus, the anti-windup not only prevents the integral action from accumulating but also prevents the control signal from moving away from the approximately linear region in the presence of sudden disturbances. Furthermore, this prevents the non-minimal phase effect from being accentuated. Figure 9 shows how this proposal improves the simulation results.
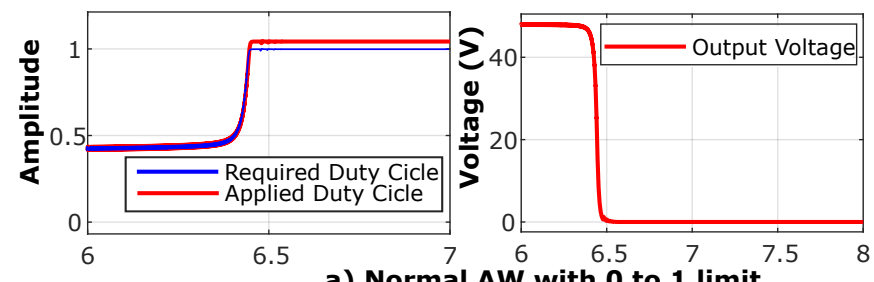

a) Normal AW with 0 to 1 limit
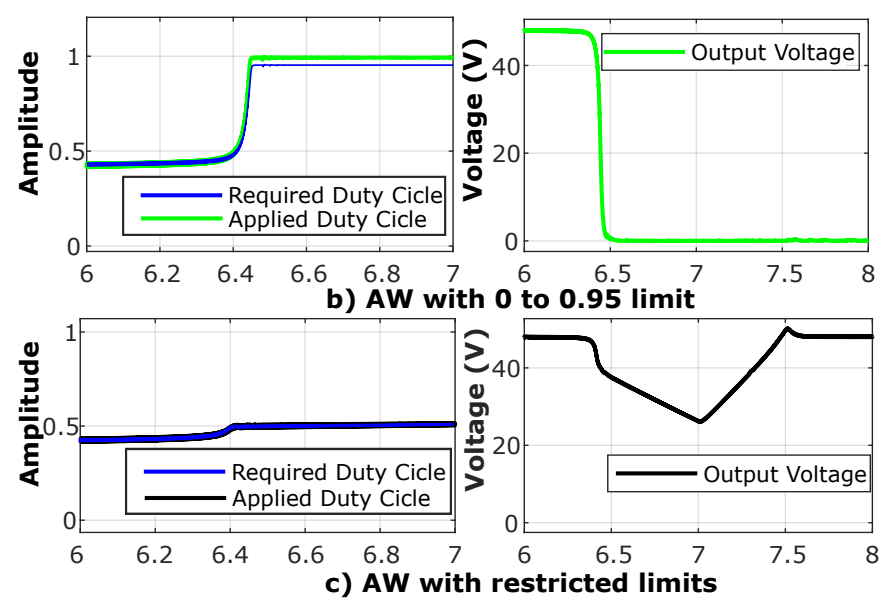

Figure 9. Anti-windup proposal strategy

This simulation set was designed purposely to present some level of disturbance at the output voltage in the buck-boost. Such instability emphasizes that apparently realistic scenarios are usually verified in Brazil due to the 


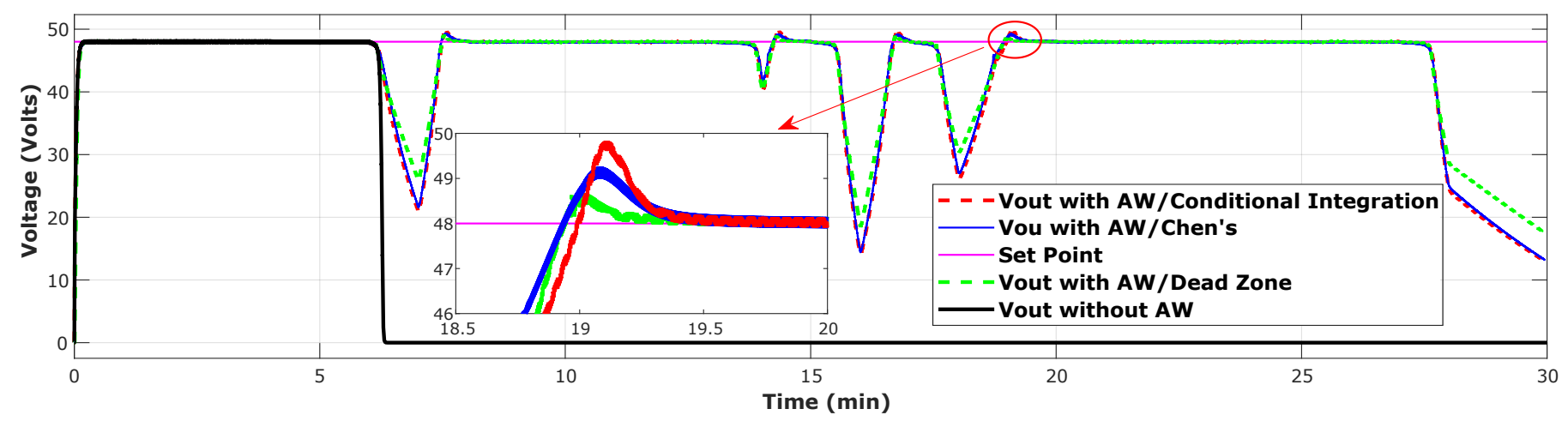

Figure 10. Output voltage of a buck-boost converter with anti-windup strategies

climate variability, and the data obtained by SONDA can be verified in multiple cutouts. Also, in the presence of disturbances in the irradiance (cloud) this type of saturation occurs naturally. Therefore, ways to mitigate this effect should be evaluated. Anti-windup techniques are simple approaches that allow for improved regulatory performance. For example, a photovoltaic system that powers a battery needs to work as a boost when the battery is discharged and, on the other hand, as a buck when the battery reaches its maximum capacity, with a risk of overheating. So, it is shown that when the irradiance reaches shallow values with abrupt variations, the input voltage of the panel drops to costs lower than $48 \mathrm{~V}$ and the buck-boost converter saturates and causes the windup phenomena, which in this case would harm the battery because the converter would not behave as required. Finally, with the insertion of the anti-windup control techniques, the converter can operate in any mode by charging or discharging with greater security and control and can mitigate the unwanted supply gap.

\section{CONCLUSIONS}

This paper has focused on comparing the behavior of a case with three anti-windup techniques subjected to saturation due to irradiance and temperature. First, the buck-boost converter was able to stabilize the output voltage, and the duty cycle with the modified conditional integrator, also with Chen's and with dead zone reach the set point. Second, Chen's performance was more sensitive to a radiation decrease and could not be able to eliminate some cloud effects if its parameter was not precisely scaled. Finally, Dead Zone, Modified Conditional Integration and Chen's strategies could improve better closed-loop performance with a PID controller, showing the relevance of its utility in the presence of irradiance and temperature effects.

Buck-Boost is typically used and is suitable for this type of application due to its versatility of raising and lowering, which allows to hold a surge as well as to increase the voltage in the presence of clouds. The big problem is that in specific cloud scenarios, only Buck-Boost will not be enough. In this context, anti-windup helps mitigate the effect of dropping on the input source (solar panel) without compromising nominal performance.

Therefore, the presented simulations evaluate the effectiveness of the proposed anti-windup techniques that can confirm, even in the presence of nonlinearities (saturation), a good disturbance rejection with a presence of clouds, a minimum steady-state error, lower overshoot and a good PWM control action which fits the duty cycle into its saturation limits.

\section{ACKNOWLEDGMENTS}

This study was financed in part by the Coordenacao de Aperfeicoamento de Pessoal de Nivel Superior - Brazil (CAPES) - Finance Code 001.

\section{REFERENCES}

Astrom, K.J. and Rundqwist, L. (1989). Integrator windup and how to avoid it.

Balamurugan, N. and Selvaperumal, S. (2016). Assessment of anti-windup pi controlled induction motor drive using $\mathrm{v} / \mathrm{f}$ method. Int $J$ Adv Engg Tech/Vol. VII/Issue I/Jan.-March, 964, 968.

Belhachat, F., Larbes, C., Barazane, L., and Kharzi, S. (2007). Commande neuro-floue d?un hacheur mppt. In 4éme conférence internationale" Computer Integrated Manufacturing", CIP, volume 7, 03-04.

Bernstein, D.S. and Michel, A.N. (1995). A chronological bibliography on saturating actuators. International Journal of robust and nonlinear control, 5(5), 375-380.

Bohn, C. and Atherton, D. (1995). An analysis package comparing pid anti-windup strategies. IEEE Control Systems, 15(2), 34-40.

Cavalcanti, M., Oliveira, K., Azevedo, G., and Neves, F. (2007). Comparative study of maximum power point tracking techniques for photovoltaic systems. Eletrônica de Potência, 12(2), 163-171.

Chen, C. (1993). Analog and Digital Control System Design. Saunders College Pub.

Dorf, R.C. and Bishop, R.H. (2011). Modern control systems. Pearson.

EIA, U. (2018). Annual energy outlook 2018. US Energy Information Administration, Washington, DC.

Erickson, R.W. and Maksimovic, D. (2007). Fundamentals of power electronics. Springer Science \& Business Media.

Fertik, H.A. and Ross, C.W. (1967). Direct digital control algorithm with anti-windup feature. ISA transactions, $6(4), 317$.

Hansson, A., Gruber, P., and Tödtli, J. (1994). Fuzzy antireset windup for pid controllers. Control Engineering Practice, 2(3), 389-396.

Hanus, R. (1980). A new technique for preventing control windup. Journal A, 21(1), 15-20. 
Hippe, P. (2006). Windup in control: its effects and their prevention. Springer Science \& Business Media.

Hodel, A.S. and Hall, C.E. (2001). Variable-structure pid control to prevent integrator windup. IEEE Transactions on Industrial Electronics, 48(2), 442-451.

Olalla, C., Leyva, R., El Aroudi, A., Queinnec, I., and Tarbouriech, S. (2009). H? control of dc-dc converters with saturated inputs. In Industrial Electronics, 2009. IECON'09. 35th Annual Conference of IEEE, 548-553. IEEE.

Rashid, M.H. et al. (2011). Power electronics handbook: Devices. Circuits and Applications, 3.

Rundqwist, L. (1990). Anti-reset windup for pid controllers. IFAC Proceedings Volumes, 23(8), 453-458.

Seghir, B., Chandra, A., and Miloud, R. (2018). A new control strategy for power quality improvement using mppt from hybrid pv-wind connected to the grid. In 2018 IEEE Canadian Conference on Electrical \& Computer Engineering (CCECE), 1-6. IEEE.

SOARES, C.E., MEZAROBA, M., MICHELS, L., and RECH, C. (2010). Técnica anti-windup para controladores $\mathrm{p}+$ ressonantes aplicados a inver-sores de tensão monofásicos com filtro lc na saída. In XVIII Congresso Brasileiro de Automática, 748-754.

SONDA (2018). http://sonda.ccst.inpe.br. Visited in $09 / 13 / 2018$.

Villalva, M., De Siqueira, T., and Ruppert, E. (2010). Voltage regulation of photovoltaic arrays: small-signal analysis and control design. IET Power Electronics, 3(6), 869-880.

Wang, Q.G., Zhang, Z., Astrom, K.J., Zhang, Y., and Zhang, Y. (2008). Guaranteed dominant pole placement with pid controllers. IFAC Proceedings Volumes, 41(2), 5842-5845.

Xiao, W., Wen, H., and Zeineldin, H. (2012). Affine parameterization and anti-windup approaches for controlling dc-dc converters. In Industrial Electronics (ISIE), 2012 IEEE International Symposium on, 154-159. IEEE.

Zheng, A., Kothare, M.V., and Morari, M. (1994). Antiwindup design for internal model control. International Journal of Control, 60(5), 1015-1024. 